\title{
Modulation of Fgfr la Signaling in Zebrafish Reveals a Genetic Basis for the Aggression-Boldness Syndrome
}

\author{
William H. J. Norton, ${ }^{1,2}$ Katharina Stumpenhorst, ${ }^{2}$ Theresa Faus-Kessler, ${ }^{3}$ Anja Folchert, ${ }^{2}$ Nicolas Rohner, ${ }^{4}$ \\ Matthew P. Harris, ${ }^{4}$ Jacques Callebert, ${ }^{5}$ and Laure Bally-Cuif ${ }^{1,2}$ \\ 1Zebrafish Neurogenetics, Laboratory of Neurobiology and Development, Institut de Neurobiologie Alfred Fessard, Unité Propre de Recherche 3294, CNRS, \\ Avenue de la Terrasse, Gif-sur-Yvette, 91190, France, ${ }^{2}$ Department of Zebrafish Neurogenetics and ${ }^{3}$ Institute for Developmental Genetics, Helmholtz Centre \\ Munich, D-85764 Munich, Germany, ${ }^{4}$ Max Planck Institute for Developmental Biology, Department of Genetics, D-72076 Tübingen, Germany, and ${ }^{5}$ Service \\ Biochimie et Biologie Moleculaire, Hopital Lariboisiere, F-75475 Paris, France
}

Behavioral syndromes are suites of two or more behaviors that correlate across environmental contexts. The aggression- boldness syndrome links aggression, boldness, and exploratory activity in a novel environment. Although aggression-boldness has been described in many animals, the mechanism linking its behavioral components is not known. Here we show that mutation of the gene encoding fibroblast growth factor receptor $1 \mathrm{a}(\mathrm{fg} f \mathrm{r} 1 \mathrm{a})$ simultaneously increases aggression, boldness, and exploration in adult zebrafish. We demonstrate that altered Fgf signaling also results in reduced brain histamine levels in mutants. Pharmacological increase of histamine signaling is sufficient to rescue the behavioral phenotype of $f g f r l a$ mutants. Together, we show that a single genetic locus can underlie the aggression-boldness behavioral syndrome. We also identify one of the neurotransmitter pathways that may mediate clustering of these behaviors.

\section{Introduction}

Many animal species exhibit behavioral syndromes (Sih et al., 2004a,b) [also called personalities (Boissy, 1995; Wolf et al., 2007), coping styles (Benus et al., 1991; Koolhaas et al., 1999), or

Received June 9, 2011; revised Aug. 2, 2011; accepted Aug. 5, 2011.

Author contributions: W.H.J.N. designed research; W.H.J.N., K.S., A.F., and J.C. performed research; N.R. and M.P.H. contributed unpublished reagents/analytic tools; W.H.J.N., T.F.-K., and L.B.-C. analyzed data; W.H.J.N. and L.B.-C. wrote the paper.

The authors declare no conflict of interest.

The Hubrecht laboratory, Sanger Institute, and MPI-CGB/CRT Dresden are supported by theZF-Models Integrated Project (Contract LSHG-CT-2003-503496 of the European Commission, respectively, to the laboratories of E. Cuppen, D. Stemple, and M. Brand). The Sanger Institute Zebrafish Mutation Resource is also sponsored by Wellcome Trust Grant WT 077047/Z/05/Z. Work in the L.B.-C. laboratory at the Helmholtz Zentrum München was funded by the Helmholtz Association, the European Union (EU) ZF-Models integrated project under Contract LSHG-CT-2003503466 and the Center for Protein Science Munich. For work at the CNRS, funds from the EU Seventh Framework Integrated Project NeuroXsys and ZF-Health, the Agence Nationale de la Recherche, Ecole des Neurosciences de Paris, Fondation pour la Recherche Médicale, and Programme Incitatif à la Mobilité d'Équipe programs, and the Schlumberger Association are gratefully acknowledged. We thank Sébastien Bedu and Michel Chaminade for assistance with fish care and behavior work and members of the Bally-Cuif laboratory for scientific comments. We thank Prisca Chapouton and Marion Coolen for discussion of the data and Monika Krecsmarik and Ina Rothenaigner for help with confocal microscopy. We are grateful for help with Nissl staining from Frank Bourrat. The spd aggression phenotype was identified by Stefanie Topp. We are grateful to Pertti Panula, Kristin Tessmar-Raible, and Michael Tsang for reagents. We thank the collaborative efforts of the Hubrecht laboratory, the Sanger Institute Zebrafish Mutation Resource, and the MPI-CGB/CRT Dresden for providing the zebrafish knock-out allele hu3264.

Correspondence should be addressed to Drs. William Norton and Laure Bally-Cuif, Zebrafish Neurogenetics, Laboratory of Neurobiology and Development, Institut de Neurobiologie Alfred Fessard, UnitéPropre de Recherche3294, CNRS, Avenue de la Terrasse, Gif-sur-Yvette, 91190, France. E-mail: norton@inaf.cnrs-gif.fr; bally-cuif@inaf.cnrs-gif.fr.

K. Stumpenhorst's present address: Molecular Neuropathology, Department of Psychiatry, University of Oxford, Oxford, 0X3 7JW, UK.

N. Rohner present address: Harvard Medical School, Department of Genetics, NRB Room 360, 77 Avenue Louis Pasteur, Boston, MA, 02115

M. P. Harris's present address: Harvard Medical School, Orthopaedic Research Laboratories, Children's Hospital, 300 Longwood Avenue, Boston, MA 02115.

DOI:10.1523/JNEUROSCI.2892-11.2011

Copyright $\odot 2011$ the authors $\quad 0270-6474 / 11 / 3113796-12 \$ 15.00 / 0$ temperaments (Réale et al., 2007)], suites of two or more behaviors that correlate across environmental contexts. Behavioral syndromes constrain the direction of evolution, affect an animal's future fitness, maintain individual differences in behavior, and may explain maladaptive behaviors (Sih et al., 2004a; Wolf et al., 2007). From an adaptive evolution point of view, the existence of behavioral syndromes is puzzling, because greater flexibility in behavior would give animals a selective advantage (Sih et al., 2004a; Bell and Sih, 2007; Wolf et al., 2007). According to the aggression-boldness syndrome, animals that are more aggressive will also be bolder and more explorative in novel environments (Huntingford, 1976). Aggression-boldness has been described in many animals, including mammals (Benus et al., 1991), birds (Dingemanse et al., 2007), and fish (Huntingford, 1976; Brick and Jakobsson, 2001; Schjolden et al., 2005; Moretz et al., 2007; Bourne and Sammons, 2008). However, the mechanism underlying its formation is not well understood and has been suggested to be caused by either pleiotropic genetic (Deng et al., 1994; Dingemanse et al., 2007) or hormonal (Koolhaas et al., 1999; Veenema et al., 2003) constraint or selection driven by optimal adaptation to the environment (Bell, 2005; Bell and Sih, 2007), with each behavioral component having a separate genetic basis.

Similar to other vertebrates, there is a correlation between aggression and boldness in zebrafish, although the relationship varies depending on the strains analyzed (Moretz et al., 2007). Zebrafish display characteristic agonistic postures, including erection of the fins, biting, tail thrashing, and short bouts of fast swimming (Gerlai et al., 2000). Aggression is exhibited by both sexes (Moretz et al., 2007) and can be influenced by both habitat complexity and rearing conditions (Basquill and Grant, 1997; Marks et al., 2005). Furthermore, different wild-type strains show 
varying aggression levels suggesting a genetic component to its control (Moretz et al., 2007). Boldness, measured as the number of approaches to a novel object, has also been shown to differ between zebrafish strains, suggesting a genetic basis to this behavior as well (Wright et al., 2003).

In this study, we examine aggression, boldness, and exploration in a zebrafish mutant with reduced fibroblast growth factor receptor 1a (Fgfrla) function, spiegeldanio (spd) (Rohner et al., 2009). Our results provide evidence that modulation of a single genetic locus, fgfrla, links the three components of the aggression-boldness behavioral syndrome, a phenotype that may be at least partly mediated through a reduction of histamine (HA) levels in the brain.

\section{Materials and Methods}

Animal strains, care, and maintenance. Adult zebrafish were maintained using standard fish-keeping protocols and in accordance with institute guidelines for animal welfare. Two days before each behavioral assay, the fish were moved to an isolated room and kept under similar conditions to minimize environmental variance and interference from background noise during testing.

Behavioral methods. Behavioral analyses were performed on 3- to 6-month-old adult Tübingen wild-type, spiegeldanio ${ }^{t 3 R 705 H}$ and spiegeldanio $^{\text {hu }}{ }^{264}$ fish of both sexes. Behavioral experiments were performed using Videotrack and LabWatcher from ViewPoint Life Sciences and a digital camera from Point Gray Research. All behavior experiments were completed between 1:00 P.M. and 4:00 P.M. to minimize circadian differences. One week before analysis, fish were habituated to the stress of being netted. Fish were daily subjected to two rounds of being caught in a fish net, held gently for $3 \mathrm{~s}$, and released into a second tank. To reduce observer bias in the manual analysis of aggressive behavior, films were quantified by two independent observers who were unaware of the genotype being analyzed. Results presented in here show the mean of both observers' findings, and the correlation between the two results was estimated using the Pearson's product-moment correlation coefficient $(r=$ 0.86). In all experiments, the age, sex, and size was carefully matched between fish of different genotypes.

Swimming behavior of fish in behavioral setups. Photographs of behavioral setups and swimming traces (see Fig. 1) were captured using the reader function in Videotrack.

Aggression. The mirror-induced aggression protocol was modified from Gerlai et al. (2000). Adult fish were maintained in groups of 20 animals from birth until the day of testing. On the day before analysis, the fish were moved to a novel $10 \mathrm{~L}$ glass fish tank. During testing, fish were placed in a small glass aquarium measuring $15 \times 10 \times 30 \mathrm{~cm}$. Both long sides and one short side of the aquarium were covered in a white opaque material. The fourth side of the tank was left clear, and a mirror was placed outside of the tank offset at an angle of $22.5^{\circ}$ (Gerlai et al., 2000). The behavior of individual fish was recorded using a high-speed digital camera set to 100 frames/s and connected to the Videotrack program. Films were manually analyzed in LabWatcher (ViewPoint Life Sciences. The results were analyzed and processed in Excel (Microsoft).

Boldness. Boldness was measured using two protocols: the time spent close to a novel object and the time spent on the nonpreferred side of a condition place-preference tank. Novel object boldness gave more reproducible results and so was used when testing the effect of histaminergic pathway drugs on both wild-type and spd fish. "Novel object boldness" was measured as the amount of time spent close to a novel object (Wright et al., 2003). The novel object was made by filling a $15-\mathrm{cm}$-long clear plastic tube with dark modeling clay. The novel object was suspended at one end of the tank, midway in the water column. Boldness was measured as the total time spent within one body length of the object in a 10 min period. "Place-preference boldness" was measured as the amount of time spent on the nonpreferred side of a place-preference tank. Fish were placed into a $10 \mathrm{~L}$ tank, the preferred side of which was colored brown and the nonpreferred side of which was white with two black spots (Ninkovic and Bally-Cuif, 2006). Fish were allowed to habituate to this setup for $2 \mathrm{~d}$. Boldness was calculated as the percentage of time spent on the nonpreferred white side of the tank. Both behavioral protocols gave similar results and so were assumed to measure the same behavior in zebrafish: boldness.

Exploration of a novel environment. Exploration was quantified as the amount of time needed to explore the arms of a novel environment. For this experiment, a T-shaped tank with one long arm and two short arms (arm lengths, $30 \times 30 \times 45 \mathrm{~cm}$ ) was used. The fish were not habituated to this setup. Fish were released into the long arm of tank, and the experiment started when they crossed a designated start line. Exploratory behavior was measured as the time taken to explore and swim within one body length of the end of each arm.

Locomotion. Locomotion was measured in a $10 \mathrm{~L}$ plastic fish tank with clear walls. Locomotion was defined as the distance swum in a 5 min period after a 10 min adjustment to the new tank.

Fish were tracked using a high-speed infrared camera with the Videotrack software set to a detection threshold of 11, inactive/small threshold of 5, and small/large threshold of 10 . The mean total distance swam (small distance and large distance) was then calculated for each animal. Locomotion was used as a readout for general behavior of the fish. Because the aggression, boldness, and exploration tests all involved swimming, a reduction in locomotion (for example, after drug treatment) would skew the behavioral results.

Olfaction. The olfactory test was modified from Vitebsky et al. (2005). Adult fish were tested for their reaction to the aversive amino acid L-serine. A glass fish tank $(15 \times 10 \times 30 \mathrm{~cm})$ was covered with white opaque material. Two flexible plastic tubes were attached to opposing corners of the tank. The tubes were connected to a $15 \mathrm{ml}$ Falcon tube filled with either saline (control) or $10^{4} \mathrm{M} \mathrm{L}$-serine. The tank was divided into two areas, and the time spent in each area was measured using Videotrack. Olfaction was quantified as the time spent away from the source of aversive L-serine.

Drug treatments. Fluoxetine hydrochloride, tacrine hydrochloride, imetit dihydrobromide, and galantamine hydrochloride were purchased from Sigma-Aldrich. Drug doses were chosen in line with those used in other species (Morisset et al., 1996). In some experiments, fish were treated with drugs before their behavior was measured. Drugs were either administered intraperitoneally $(1 \mathrm{mg} / \mathrm{g}$ tacrine hydrochloride, imetit dihydrobromide, or galantamine hydrochloride, once per day for $3 \mathrm{~d}$; Sigma-Aldrich) or directly into the tank water ( $3 \mu \mathrm{g} / \mathrm{ml}$ fluoxetine hydrochloride for 3 h; Sigma-Aldrich) (Lynn et al., 2007). For control experiments, animals were treated with saline instead of drug.

Histochemistry reagents. In situ hybridization was performed according to Norton et al. (2008) and antibody staining according to Topp et al. (2008). The following in situ hybridization probes were used in this study: dusp6; fgfr1a; mao; npvf; pomcb; slc6a4a; and slc6a4b. For gene information, refer to www.zfin.org. Histamine $n$-methyl transferase $(h n m t)$ was amplified using the primers GGCTCAATGACTAAGATTCA AG and GCTCCATTCAAAACACTTGT. hnmt was cloned into the StrataClone PCR Cloning Vector $p S C$-A (Stratagene). The following antibodies were used: anti-phosphorylated extracellular signal-regulated kinase (pERK) [mitogen-activated protein kinase (MAPK); 1:1000; Sigma], anti- proliferating cell nuclear antigen (PCNA) (1:1000; Dako), anti-gonadotrophin releasing hormone (GnRH) (1:500; Sigma), and anti-histamine (1:2000; gift from Dr. P. Panula, University of Helsinki, Helsinki, Finland). TUNEL staining was performed using the Neurotacs 2 in situ apoptosis detection kit (Trevigen). Specimens were photographed with an Axioplan2 stereomicroscope for in situ staining (Carl Zeiss) or Axioplan2 ApoTome for antibody labeling (Carl Zeiss). Figures were mounted in Adobe Photoshop version CS2 (Adobe Systems). Nissl staining was performed on cryosections of the brain according to standard protocols.

Radioimmunoassay. Whole adult brains were snap frozen in liquid nitrogen and stored at $-80^{\circ} \mathrm{C}$ until processing. Brains were homogenized by grinding up in $150 \mu \mathrm{l}$ of $0.2 \mathrm{~N} \mathrm{HCLO}_{4}$. After centrifugation at $10,000 \times g$ for $5 \mathrm{~min}$ at $4^{\circ} \mathrm{C}$, the supernatant was neutralized to $\mathrm{pH} 6-8$ by addition of an equal volume of $1 \mathrm{~m}$ potassium borate and then passed through a $10 \mathrm{kDa}$ filter (Nanosep $10 \mathrm{kDa}$; Pall Life Sciences, VWR). Two aliquots of $100 \mu \mathrm{l}$ 
were analyzed for histamine levels by radioimmunoassay (RIA Histamine kit, Immunotech), following the instructions of the manufacturer.

ELISA. Blood plasma was collected from pools of three male and three female adult fish (each yielding between 1 and $5 \mu \mathrm{l}$ of blood, to make a total of $25 \mu \mathrm{l}$ ). Fresh blood was mixed $1: 10$ with a citrate buffer and stored in heparinized hematocrit tubes. Blood samples were centrifuged at $4^{\circ} \mathrm{C}$ for $10 \mathrm{~min}$, and the plasma was aspirated. ELISA was performed using either a testosterone immunoassay kit (DNOV002) or a $17 \beta$-estradiol immunoassay kit (DNOV001) from AbCys.

Real-time PCR. Quantitative PCR analysis (qPCR) was performed by Eurofins. Primers were designed and optimized by Eurofins (qPCR primer sequences available on request). PCRs were performed on an ABI 7900 SDS machine using a SYBR Green Universal Master Mix (Exiqon). cDNA was synthesized from 1 $\mu \mathrm{g}$ of RNA extracted from whole brains. The PCR conditions were $56^{\circ} \mathrm{C}$ for $10 \mathrm{~min}, 94^{\circ} \mathrm{C}$ for $10 \mathrm{~min}$, followed by 40 cycles of $60^{\circ} \mathrm{C}$ for $1 \mathrm{~min}$ and $94^{\circ} \mathrm{C}$ for $15 \mathrm{~s}$. Analysis was performed in duplicate using 10 biological replicates for each genotype. All results were separately normalized to the expression level of four housekeeping genes: gapdh, mapta, neurod, and tubb2b. Because all housekeeping genes showed similar results, only data from neurod are presented in this study. qPCR results are shown as an expression ratio calculated relative to neurod. Relative expression levels were calculated using the arithmetic comparative method, $\left(2^{-\Delta \Delta \mathrm{Ct}}\right)$, which includes a correction between $E$ (PCR amplification efficiency) for the target and housekeeping gene (Livak and Schmittgen, 2001). Results were expressed as relative expression ratios (REs) using neurod as a reference gene in the formula $\mathrm{RE}=(E \text { ref })^{\mathrm{Ct} \text { ref }}$ / ( $E$ target $)^{\mathrm{Ct} \text { target }}$, where Ref is the housekeeping gene, target is the gene of interest, and $\mathrm{Ct}$ is the crossing point (Filby and Tyler, 2005). For statistical analysis, the Student's $t$ test was performed with $n=10$ for both genotypes.

$q P C R$ primer sequences. Primer sequences used for quantitative real-time PCR analysis of neurotransmitter encoding gene expression in $s p d$ and wild-type brains included the following: $h d c$ forward, TCCAGCAGTAATCATCCA CTCGT; $h d c$ reverse, TTAGAGGAGCGAGG CGTCTC; $h n m t$ forward, GAAAGAGTTCAG TAAAAATGCTCCTGA; $h n m t$ reverse, ATAT GATCCTGCCGTCCACC; $h r h 1$ forward, GA AACCTGCGTCCATCACG; $h r h 1$ reverse, AA GTTTTCGTTGCAGAGCGG; $h r h 2$ forward, CA CGCCCATTCTTCAAAAGG; $h r h 2$ reverse, CC ATTTGTAGCGCTGTTATCGTC; $h r h 3$ forward, TTCTGGCTGCTGTGGATCAA; and $h r h 3$ reverse, TTGCTCGGACACAGTAATTTGC.

Statistics. All error bars denote SEM. For aggression, boldness, exploration, locomotion, cell counts, and qPCR analysis, we used Welch's twosample $t$ test allowing for unequal variances. For olfactory behavior, fluoxetine hydrochloride, tacrine hydrochloride, imetit dihydrobromide, and galantamine hydrochloride treatment data were analyzed by two-way ANOVA with genotype and a

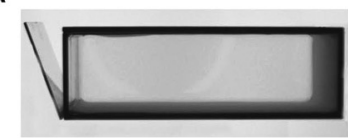

b

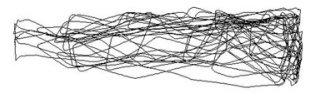

WT C

spd d

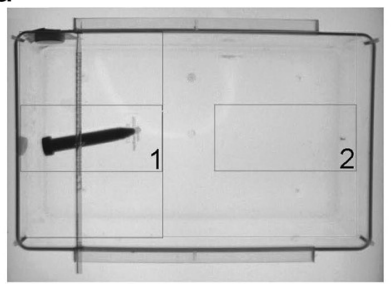

g

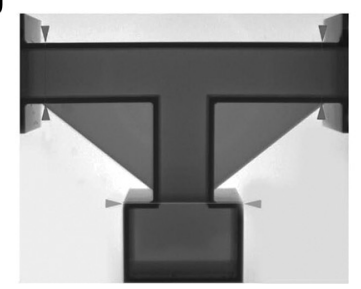

j

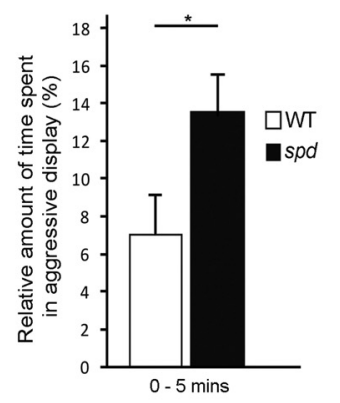

I

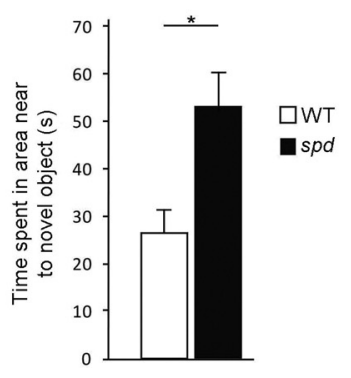

e

WT f

spd

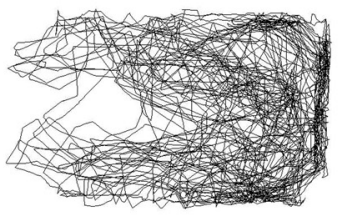

$\mathrm{h}$

WT i

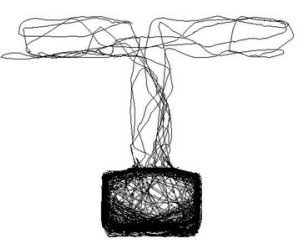

spd

k

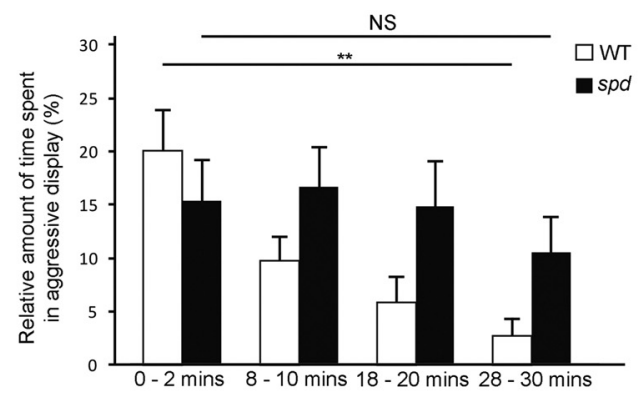

$\mathrm{m}$

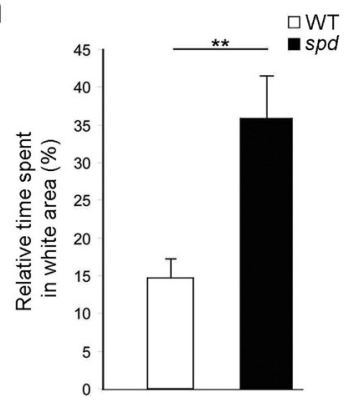

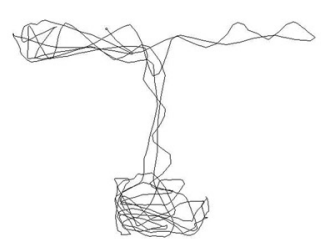

spd

n

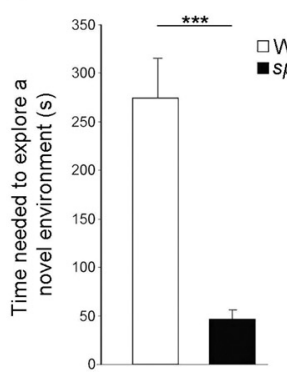

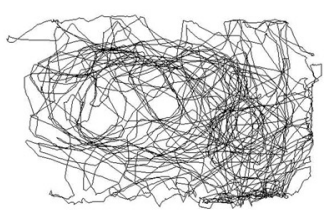

Figure 1. spiegeldanio mutants are more aggressive, bolder, and more explorative than wild-type fish. $\boldsymbol{a}$, Photograph of tank used to measure aggressive behavior (top view). A mirror is placed on the left side of the setup, offset at an angle of $22.5^{\circ}$. $\boldsymbol{b}$, Representative trace of wild-type swimming behavior in the aggression setup. $\boldsymbol{c}$, Representative trace of spd swimming behavior in the aggression setup. $\boldsymbol{d}$, Photograph of tank used to measure novel-object boldness (top view). The novel object is seen on the left side of the tank. Time spent in area 1 (near to a novel object) and area 2 (a control area away from the object) was compared. $\boldsymbol{e}$, Representative trace of wild-type swimming behavior in the novel-object boldness setup. $\boldsymbol{f}$, Representative trace of spd swimming behavior in the novel-object boldness setup. $\boldsymbol{g}$, Photograph of tank used to measure exploration (top view). The time needed to swim within one body length of the two ends of the tank (shown by red lines and red arrowheads) after crossing an initial start line (green line and green arrowheads) was recorded. $\boldsymbol{h}$, Representative trace of wild-type swimming behavior in the exploration setup. $\boldsymbol{i}$, Representative trace of spd swimming behavior in the exploration setup. $\boldsymbol{j}$, spd mutant fish (black bar) are more aggressive than wild-type siblings (white bar). $p=0.04 ; n=12$ wild-type and $n=10$ spd. $t$ test. Interaction with a mirror image for 5 min. $\boldsymbol{k}$, spd mutants maintain stable aggression levels (black bars), whereas wild-type fish (white bars) serially reduce aggression over time. Fish were exposed to a mirror image for $30 \mathrm{~min}$, and behavior was recorded during $2 \mathrm{~min}$ time intervals. Wild-type $0-2 \mathrm{~min}$ versus wild-type $28-30$ min: $p=0.02 ; n=7$ wild-type. $t$ test. $s p d 0-2$ min versus spd $28-30$ min: $p=0.6 ; n=7$ spd. $t$ test. $I$, spd mutants (black bar) are bolder than wild-type siblings (white bar), spending more time near to a novel object. $p=0.04 ; n=$ 19 wild-type and $n=17$ spd. $t$ test. $\boldsymbol{m}$, spd mutants (black bar) are bolder than wild-type siblings (white bar), spending more time on the nonpreferred side of a place-preference tank. $p=0.008 ; n=8$ wild-type and $n=8$ spd. $t$ test. $n$, spd mutants (black bar) explore a novel aquarium faster than wild-type siblings (white bar). $p=0.0003 ; n=10$ wild-type and $n=10$ spd. $t$ test. 
drug. First, a model with interaction was fitted. If there was a significant interaction effect, the genotype difference was tested in the subgroups of drug treatment. If the interaction term was not significant, a two-way ANOVA without interaction was fitted. If appropriate, post hoc comparisons were performed by $t$ tests with $p$ value adjustment for multiple testing according to Holm (1979). When necessary, data were log-transformed to normalize the distribution. Statistical significance was depicted as follows: NS (nonsignificant), $p>0.05 ;{ }^{*} p<0.05,{ }^{* *} p<0.01,{ }^{* *} p<0.001$. In all cases the number of animals tested is denoted by $n$.

\section{Results}

Reduction of Fgfr la increases aggression in zebrafish

During routine stock keeping, we noticed that spiegeldanio [fgfr $1 a^{t 3 R 705 H / t 3 R 705 H}$, also called spd (Rohner et al., 2009)] mutants showed an increased tendency to kill other fish and to bite conspecifics during pairing. To quantify these observations, we measured agonistic behavior by using mirror-image stimulation (Rowland, 1999; Gerlai et al., 2000; Clotfelter et al., 2007). Fish are unable to recognize their own mirror image and thus attack as if an intruder is present (Rowland, 1999). Homozygous spd mutants were clearly more aggressive than siblings during a $5 \mathrm{~min}$ period (Figs. $1 a-c, j, 2 a$ ). Whereas wild-type fish serially reduced aggression levels after initiation, spd mutants maintained high levels of agonistic behavior throughout the experiment, failing to downregulate aggression over time (Fig. 1k). Agonistic behavior was not sex specific, in agreement with other studies of zebrafish aggression (Moretz et al., 2007) (Fig. 2b). We therefore used fish of both sexes in our subsequent behavioral analyses.

To confirm that alteration of $f g f r l a$ activity underlies the observed behavioral defects, we measured aggression levels in fish mutant for a second $f g f r 1 a$ allele, $f g f r 1 a^{h u 3264}$. Both $f g f r l a$ alleles harbor strong hypomorphic mutations in the kinase domain of fgfrla (Rohner et al., 2009) (Fig. 3a). However, hu3264 shows variable penetrance and juvenile lethality as homozygotes, suggesting that it causes a more severe reduction of $f g f r l a$ activity than the t3R705H allele (Rohner et al., 2009). The lethality of juvenile fgfr1 $a^{\text {hu3264/hu3264 }}$ fish precluded their use in adult behavioral experiments. We thus quantified aggression in transheterozygous fish $\left(f g f r 1 a^{t 3 R 705 H / h u 3264}\right)$. Like homozygous $s p d^{t 3 R 705 H / t 3 R 705 H}$, transheterozygous fgfr $1 a^{t 3 R 705 H / h u 3264}$ fish showed a significant increase in aggression compared with siblings (Fig. 2c). Fish heterozygous for either allele showed a nonsignificant dominant trend increase in aggression compared with wild type, suggesting that a gene dosage effect may exist for this phenotype. We decided to use $f g f r 1 a^{t 3 R 705 H / t 3 R 705 H}$ in our subsequent experiments (hereafter referred to as homozygous spd mutants).

\section{spiegeldanio mutants exhibit hallmarks of the aggression-boldness syndrome}

Because an aggression-boldness syndrome has been observed in some zebrafish strains (Moretz et al., 2007), we surmised that the increased aggression levels in homozygous spd might be paralleled by increases in boldness and exploratory activity. Boldness was quantified by using two behavioral assays. We first measured the amount of time spent close to a novel object [novel-object boldness(Wright et al., 2003)]. Neither wild-type nor homozygous spd fish showed a preference for either side of an empty boldness tank (Fig. $2 f$ ) or a difference in the distance swum in the presence of the novel object (Fig. $2 g$ ). However, homozygous spd mutants spent significantly more time within one body length of the novel object than wild-type fish (Fig. $1 d-f, l$ ), suggesting an increase in boldness. The second boldness paradigm recorded the amount of time spent on the nonpreferred side of a biased place-
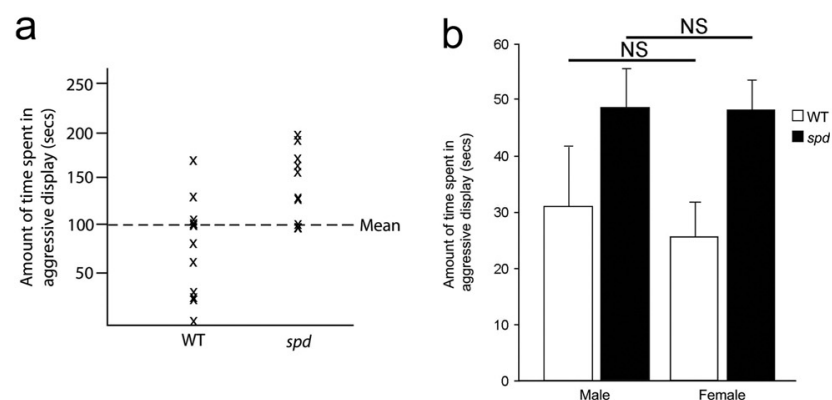

C
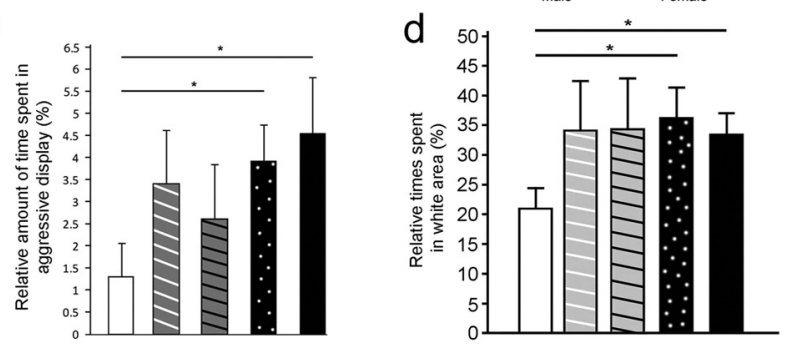

$\square$ fgfria $+/+$ 曰 fgfr1a t3R705H/+ 目 fffr1a hu3264/t+

Df ffria t3R705H/huз264

- fgfr1a t3R705H/t3R705H

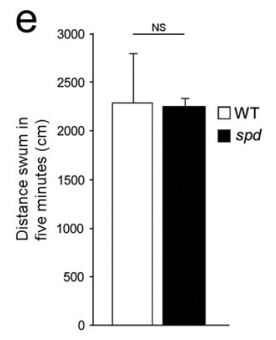

f $\square$ fgfr1a+/+ $\boxminus$ fgfr1a $t 3 R 705 \mathrm{H} /+$ 目 fgria hu3264/+ - fgfria 3 R $705 \mathrm{H} / \mathrm{hu} 3264$ - fgfr1a 3 R $705 \mathrm{H} / \mathrm{t} 3 \mathrm{R} 705 \mathrm{H}$

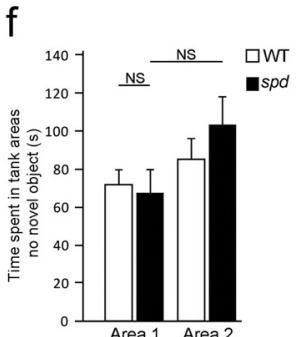

g

Figure 2. Control behavioral experiments. $\boldsymbol{a}$, Aggression levels overlap in both wild-type and spd. Each X represents one fish. $n=12$ wild-type and $n=11$ spd. Interaction with a mirror image for $10 \mathrm{~min} . \boldsymbol{b}$, Males and females of both genotypes show similar aggression levels. Males wild-type versus spd, $p=0.6$ and female wild-type versus spd, $p=0.2 ; n=10$ males and $n=$ 10 females per genotype. $t$ test. c, Both transheterozygous ( fgfr $1 a^{t 3 R 705 H} / \mathrm{fgfr} a^{\text {hu } u 264}$; black spotted bar, $p=0.03$ ) and homozygous ( fgfr $1 a^{t 3 R 705 H} / f_{f f r} 1 a^{t 3 R 705 H}$; black bar, $p=0.04$ ) spiegeldanio mutant fish are more aggressive than wild-type siblings (white bar). Heterozygous fish are not significantly more aggressive than wild-type ( fgfr $1 a^{t 3 R 705 \mathrm{H} /+}$; white hatches on gray bar, $p=0.35$ and fgfr $1 a^{\text {hu3264/+ }}$; black hatches on gray bar, $p=0.38$ ); $n=9$ wild-type, $n=14$ fgfr $1 a^{\text {t3R705/+ }}, n=9$ fgfr $1 a^{h \text { hu3264/+ }}, n=10$ fgfr $1 a^{t 3 R 705 H} / / f g f r 1 a^{h u 3264}$, and $n=9$ fgfr $7 a^{t 3 R 705 H / t 3 R 705 H}$. $t$ test. Aggression measured as the interaction with a mirror image for 10 min. $\boldsymbol{d}$, Both transheterozygous ( $\mathrm{fgfr} 1 a^{t 3 R 705 \mathrm{H}} / \mathrm{fgfr} 1 a^{\text {hu3264}}$; black spotted bar, $p=0.03$ ) and homozygous ( fgfr $1 a^{t 3 R 705 H} / f_{\text {ffrr }} 1 a^{t 3 R 705 H}$; black bar, $p=0.03$ ) spiegeldanio mutant fish are bolder than wild-type siblings (white bar). Heterozygous fish are not significantly bolder than wild-type ( fgfr $1 a^{t 3 R 705 H /+}$; white hatches on gray bar, NS, $p=0.11$ and fgfr $1 a^{\text {hu3264/+ }}$; black hatches on gray bar, $p=0.18) ; n=9$ wild-type, $n=11$ fgfr $1 a^{t 3 R 705 /+}, n=9$ fgfr $1 a^{\text {huz264/+ }}$,

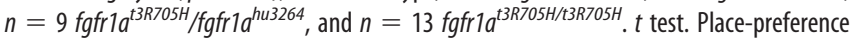
boldness assay. $\boldsymbol{e}$, Both spd (blackbar) and wild-type (white bar) show similar locomotion levels in a familiar environment. $p=0.93 ; n=10$ wild-type and $n=10$ spd. $t$ test. $\boldsymbol{f}$, Neither spd (black bars) nor wild-type fish (white bars) show a preference for either side of an empty novel object boldness tank. For depiction of tank areas, refer to Figure $1 d$. Area 1 versus area $2, F=$ $0.31, p=0.6$; wild-type versus spd, $F=3.41, p=0.07 ; n=7$ wild-type and $n=7$ spd. Two-way ANOVA without interaction. $\boldsymbol{g}$, Both wild-type (white bar) and spd (black bar) fish swim a similar distance in the presence of a novel object. $p=0.07 ; n=12$ wild-type and $n=$ 10 spd. $t$ test.

preference tank [place-preference boldness(Ninkovic and BallyCuif, 2006)]. Wild-type fish spent on average $14.7 \%$ of the time on the nonpreferred (frightening) side compared with $35.9 \%$ of time spent by homozygous spd mutants (Fig. $1 \mathrm{~m}$ ). The similar results of both assays (place preference and reaction to a novel 
object) support the idea that they measure the same behavior in zebrafish. Together, the results of both these tests suggest that spd exhibits enhanced boldness compared with siblings. Similar to aggression levels, fish heterozygous for either allele show a nonsignificant trend increase in boldness, and both spd and transheterozygous fish show significantly increased boldness behavior (Fig. $2 d$ ).

We next analyzed exploratory activity in a novel environment, a T-shaped aquarium. We recorded the amount of time needed to swim within one body length of each end of the tank. Homozygous spd mutant fish explored this environment in significantly less time than wild-type siblings (Fig. $1 g-i, n$ ), thereby showing increased exploration. However, homozygous spd mutants did not exhibit changes in locomotion compared with wild type (Fig. 2e). Together, these results suggest that reduction of Fgf signaling in spd results in concomitant increases in aggression-boldness.

In humans, autosomal dominant lossof-function mutations in FGFR1/KAL2 cause Kallmann syndrome (KS), which comprises both anosmia/hyposmia and hypogonadotropic hypogonadism caused by a reduction of GnRH levels and hypoplasia of the olfactory bulb and tract (Dodé et al., 2003). KS is a developmental disease with highly variable penetrance; patients with the same mutation within FGFR1 show a range of symptoms, from normosmia and fertility to complete KS. Because spd codes for one of the two zebrafish orthologs of FGFR1/KAL2 (Fig. $3 a$ ), we asked whether homozygous spd mutants share phenotypic similarities with KS. We found that homozygous spd are able to detect the aversive amino acid L-serine (Vitebsky et al., 2005) (Fig. 3b) and so have at least some ability to detect odors. Homozygous spd also have no changes in the expression of $\mathrm{GnRH}$ in the preoptic area (Fig. 3c) and similar blood levels of testosterone and $17 \beta$-estradiol as wild-type fish (Fig. 3e). Therefore, homozygous spd mutants do not share obvious phenotypes with KS patients.

$s p d$ mutants have a major reduction of Fgf signaling in the inferior

lobe of the hypothalamus

Compared with mice and medaka deficient in $f g f r 1$ activity (Deng et al., 1994; Yokoi et al., 2007), homozygous spd mutants have a surprising lack of early developmental phenotype, perhaps because of the presence of two paralogous $f g f r 1$ genes with similar expression patterns during development (Rohner et al., 2009). However, only $f g f r 1 a$ is expressed in the adult zebrafish brain, and the expression of $f g f r l a$ is globally similar in both wild-type and
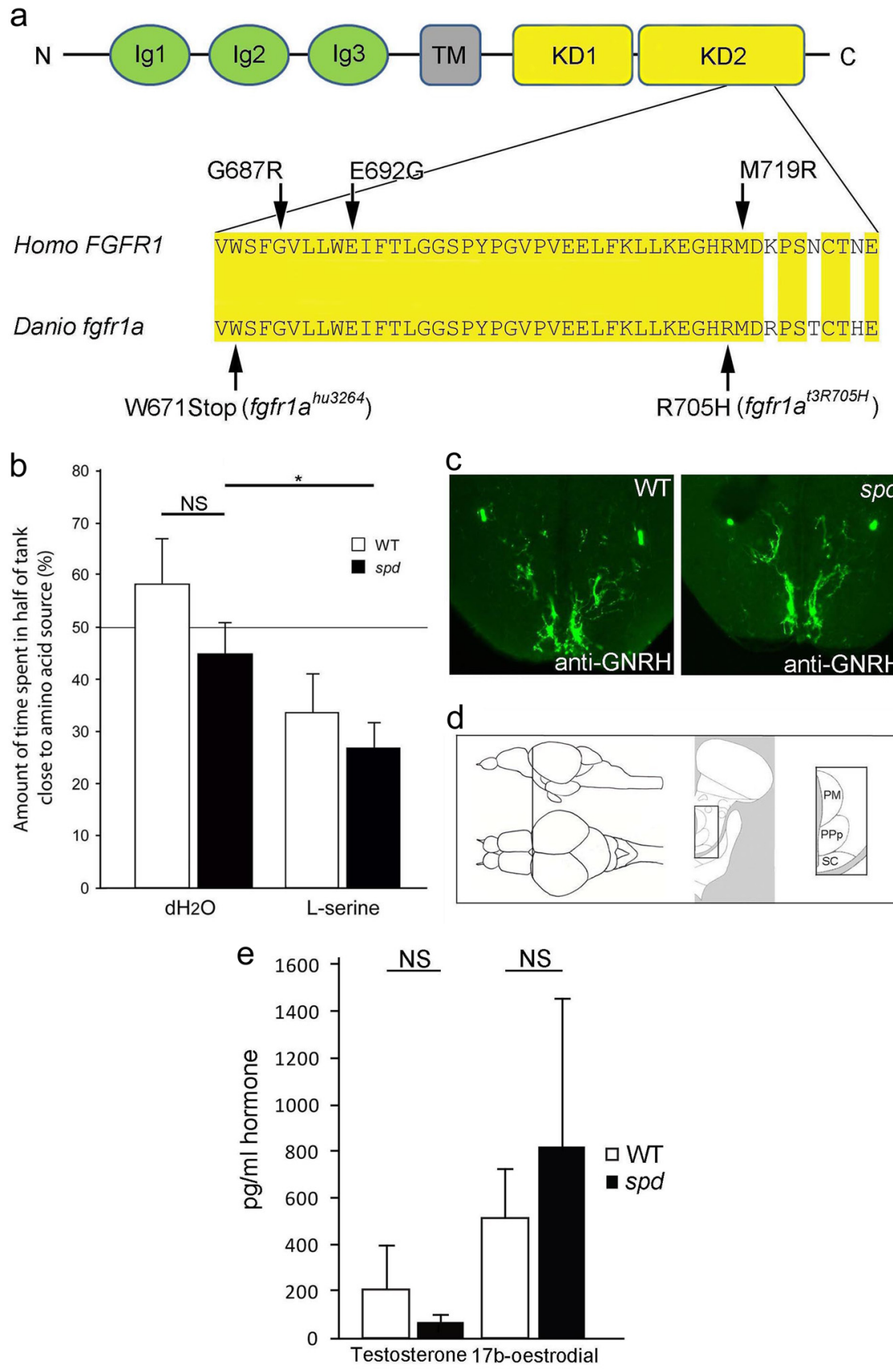

Figure 3. spd mutants do not show a Kallmann syndrome-like phenotype. $\boldsymbol{a}$, Diagram showing the position of mutations in the human FGFR1A (G687R, E692G, M719R) and zebrafish [hu3264 (W671Stop), spd (t3R705H)] Fgfr1a protein. Mutations are not conserved between species. $\boldsymbol{b}$, Mutation of fgfr 1 a does not cause olfactory defects in zebrafish. Both wild-type (white bars) and spd (black bars) mutants are averse to the smell of the amino acid $\mathrm{L}$-serine but do not react to water. Wild-type versus spd, $F=1.615$, $p=0.208$; L-serine versus $\mathrm{dH} 20, F=8.606, p=0.006 ; n=9$ wild-type and $n=8 \mathrm{spd}$. Two-way ANOVA without interaction. $c$, Anti-gonadotrophin releasing hormone antibody labeling is similar in the anterior hypothalamus of both wild-type and spd. $\boldsymbol{d}$, Diagram depicting the position of sections in c in the zebrafish brain. PM, Magnocellular preoptic nucleus; PPp, posterior part of the parvocellular preoptic area; $S C$, suprachiasmatic nucleus. $\boldsymbol{e}$, There is no significant difference in levels of testosterone and $17 \beta$ estradiol in the blood of wild-type (white bars) and spd (black bars). Wild-type versus spd testosterone, $p=0.7$; wild-type versus spd $17 \beta$-estradiol, $p=0.67 ; n=18$ wild-type and $n=18$ spd. $t$ test.

mutant fish. To examine the Fgf signaling defect in spd, we analyzed expression of the dual specificity phosphatase enzyme dusp6 (Fig. 4a,b) and pERK (Fig. $4 c, d$ ), readouts of Fgf signaling in the adult brain (Topp et al., 2008). We observed a reduction of both $d u s p 6$ and pERK in the periventricular nucleus of the inferior lobe of the hypothalamus (PVN; Fig. $4 a-d, k$ ). However, other brain areas, such as the central nucleus of the hypothalamus 

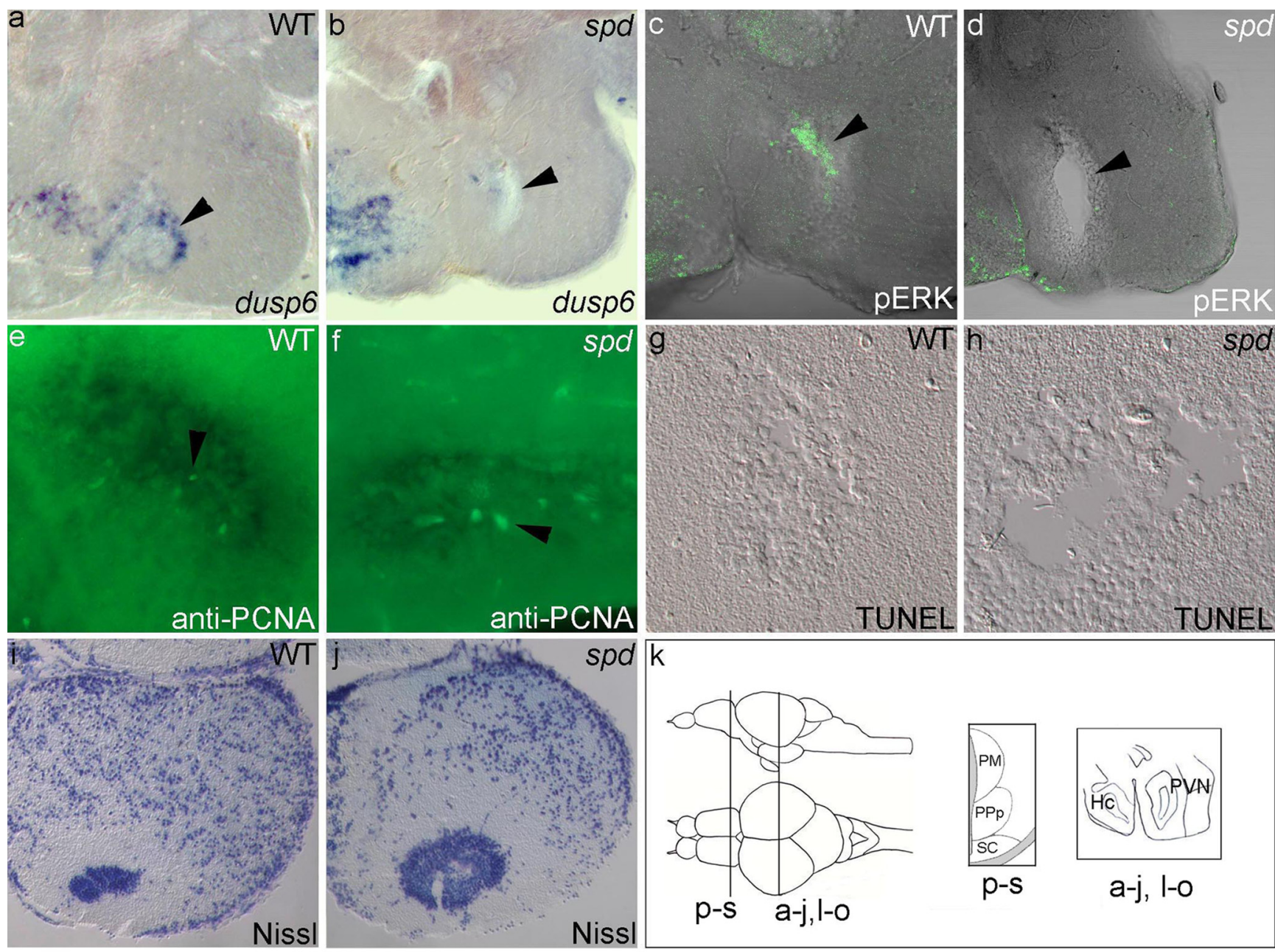

p-s

a-j, I-O
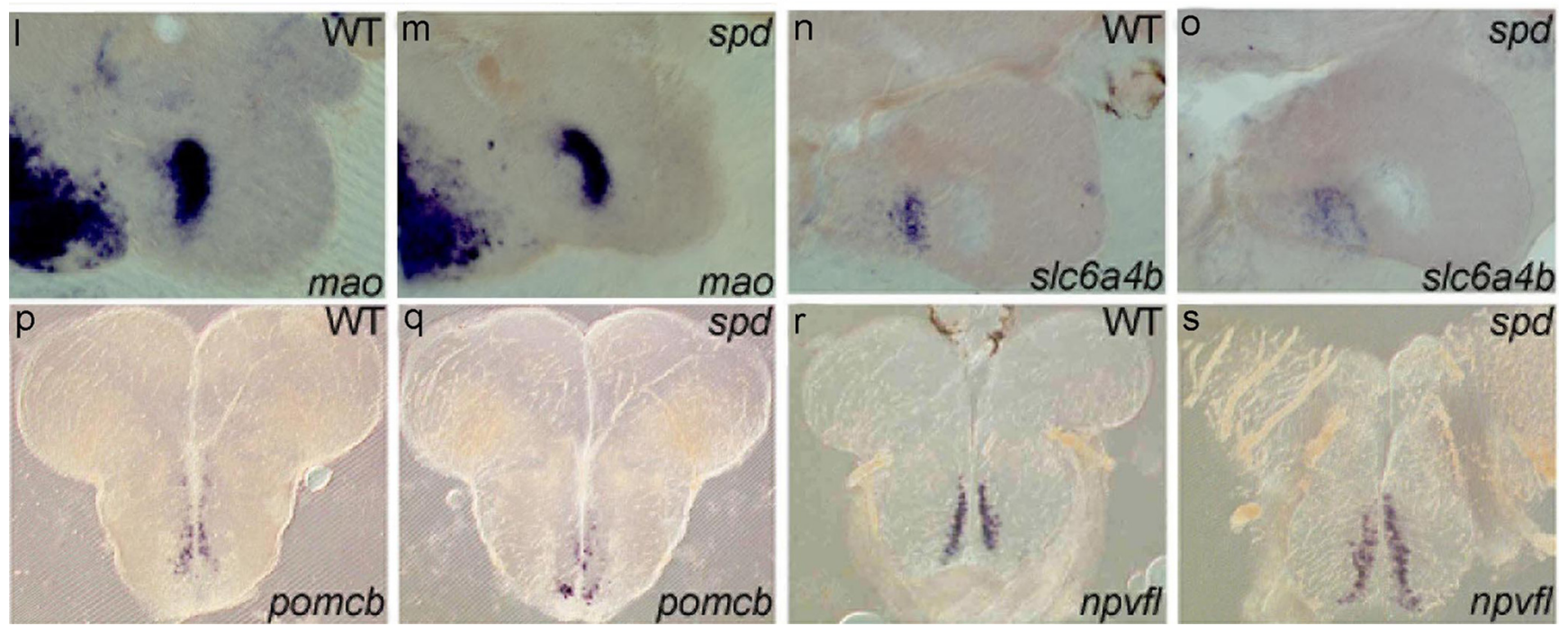

Figure 4. spd have a reduction of Fgf signaling in the periventricular nucleus. $\boldsymbol{a}-\boldsymbol{j}$, High-magnification images of coronal sections through the adult zebrafish hypothalamus, showing dusp 6 in situ hybridization $(\boldsymbol{a}, \boldsymbol{b})$, pERK immunocytochemistry $(\boldsymbol{c}, \boldsymbol{d})$, PCNA immunocytochemistry $(\boldsymbol{e}, \boldsymbol{f})$, TUNEL labeling $(\boldsymbol{g}, \boldsymbol{h})$, and Nissl staining $(\boldsymbol{i}, \boldsymbol{j})$. dusp6 and pERK are reduced in the PVN of spd $(\boldsymbol{b}, \boldsymbol{d})$ compared with siblings $(\boldsymbol{a}, \boldsymbol{c})$. PCNA and TUNEL labeling is similar between wild-type $(\boldsymbol{e}, \boldsymbol{g})$ and spd $(\boldsymbol{f}, \boldsymbol{h})$. The Nissl-stained PVN of spd $(\boldsymbol{j})$ appears larger than wild-type $(\boldsymbol{i})$. Arrowheads in $\boldsymbol{a}$ - $\boldsymbol{d}$

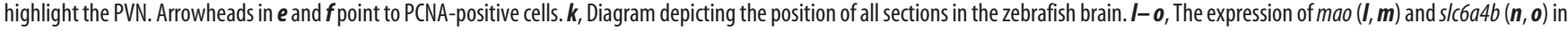
the periventricular nucleus of both wild-type $(\boldsymbol{I}, \boldsymbol{n})$ and spd $(\boldsymbol{m}, \boldsymbol{0})$. Expression levels are similar in both genotypes. $\boldsymbol{p}$-s, The expression of pomcb $(\boldsymbol{p}, \boldsymbol{q})$ and $n v p f(\boldsymbol{r}, \boldsymbol{s})$ in the preoptic area of both wild-type $(\boldsymbol{p}, \boldsymbol{r})$ and spd $(\boldsymbol{q}, \boldsymbol{s})$. Expression levels are similar in both genotypes. PM, magnocellular preoptic nucleus; PPp, parvocellular preoptic nucleus posterior part; $\mathrm{SC}$, suprachiasmatic nucleus. 
(Hc), were essentially unaffected (Fig. $4 a-$ $d, k)$. During closer inspection, the ventricle surrounded by the PVN appears larger in homozygous spd (Fig. 4j) than wild type (Fig. 4i), supporting our observation that Fgf function is altered in this region. However, analysis of anti-PCNA antibody (marking dividing cells; Fig. 4e,f) and TUNEL staining for apoptosis (Fig. $4 g, h$ ) did not reveal addition defects between wild-type and mutant hypothalami. We examined more genes expressed in the $\mathrm{Hc}$ and PVN (Fig. $4 l-o$ ). All of the genes that we analyzed were similarly expressed in the inferior hypothalamus of both genotypes, indicating that the PVN is not globally reorganized. Thus, homozygous spd mutants have a major reduction of Fgf signaling in the PVN.

We next expanded our characterization of the mutant brain to include other neural markers. The preoptic area of the anterior hypothalamus (POA) is another area of the zebrafish brain implicated in the control of aggression and dominance (Larson et al., 2006). In contrast to the $\mathrm{PVN}$, the POA looked morphologically normal, and we found similar expression of POA marker genes in both wild-type and homozygous spd (Fig. $4 p-s$ ).

\section{Behavioral alterations in spd are caused by reduced histaminergic neurotransmitter signaling}

We next aimed to identify the neurotransmitter pathways linking Fgf signaling and behavior. 5-HT acting via Htrla (Gross et al., 2002; Clotfelter et al., 2007) and Htr1b receptors (Ramboz et al., 1996) has been identified as having a key role in the control of aggression and boldness/anxiety in fish and mouse. Treatment of fish with the selective 5-HT reuptake inhibitor fluoxetine hydrochloride increases 5-HT levels and has been shown to reduce aggression levels in fish (Perreault et al., 2003; Lynn et al., 2007). We first observed an increase in the number of neurons expressing the 5-HT transporter gene slc6a4a in the raphe nucleus of $s p d$ (Fig. $5 a, b, d$ ).

To assess the contribution of 5-HT signaling to the behavior of $s p d$, we gave fish an acute nonsedative (see Fig. $8 a$ ) dose of fluoxetine and measured subsequent changes to aggression, boldness, and exploration. Surprisingly, although fluoxetine reduced aggression levels in both genotypes, it failed to blunt the increased aggression in $s p d$ (Fig. 5e). We next measured place-preference boldness and exploration. Modifying 5-HT signaling with fluoxetine caused a significant increase in the place-preference boldness levels of both wild-type and homozygous spd without rescuing the mutant behavior (Fig. $5 f$ ); thus, similar to the aggressive behavior, 5 -HT neurotransmission does not underlie the increased boldness in spd. Conversely, fluoxetine treatment did not significantly alter exploratory activity in either homozygous spd or wild type (Fig. 5g), ruling out a role for 5-HT signaling in this behavior. We thus conclude that
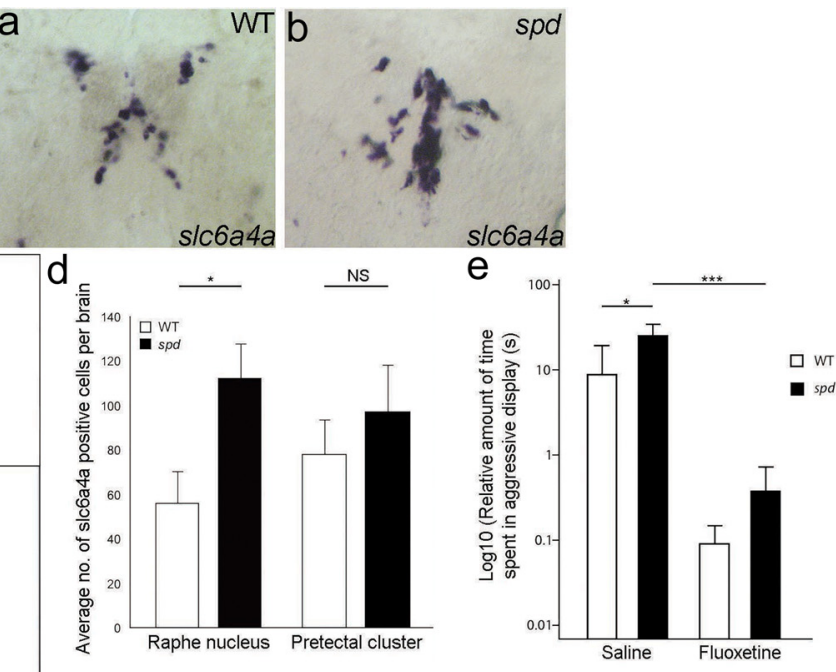

Figure 5. 5-HT signaling does not underlie the spd behavioral phenotype. $\boldsymbol{a}, \boldsymbol{b}$, High-magnification images of coronal sections 列 mutants (black bars) with or without fluoxetine treatment. wild-type versus spd, $F=2.134, p=0.04$; fluoxetine versus (measured in place-preference tank) in spd. Compare wild-type fish (white bars) with spd mutants (black bars) 作 10 wild-type saline, $n=13$ spd saline, $n=12$ wild-type fluoxetine, and $n=12$ spd fluoxetine. Two-way ANOVA without , Fluoxetine treatment does not influence exploration in either wild-type or spd. Compare wild-type fish (white bars) versus saline, $F=0.239, p=0.548 ; n=8$ wild-type saline, $n=8$ spd saline, $n=9$ wild-type fluoxetine, and $n=10$ spd fluoxetine. Two-way ANOVA without interaction.

5-HT signaling does not underlie the Fgfrla-dependent aggression-boldness syndrome. However, it is possible that the increase in slc6a4a expression seen in the dorsal raphe nucleus of mutants may be connected to the behavioral changes that we observe in a 5-HT-independent manner.

To broaden our analysis to include other neurotransmitter pathways, we used qPCR to examine the expression of $40 \mathrm{com}$ ponents of the dopaminergic, 5-HTergic, noradrenergic, and histaminergic neurotransmitter pathways, including synthesis enzymes, receptors, and transporters. The majority of these genes showed no change in expression, whereas the gene encoding $h n m t$ was significantly upregulated in homozygous spd mutants compared with wild type (Fig. 6a), representing a 1.06-fold change in mRNA levels. hnmt curbs histaminergic signaling in the brain by converting $\mathrm{HA}$ to its metabolite $\mathrm{N}$-methylhistamine (Haas et al., 2008). hnmt is widely expressed throughout the brain, with similar distribution in both wild type and spd (Fig. 

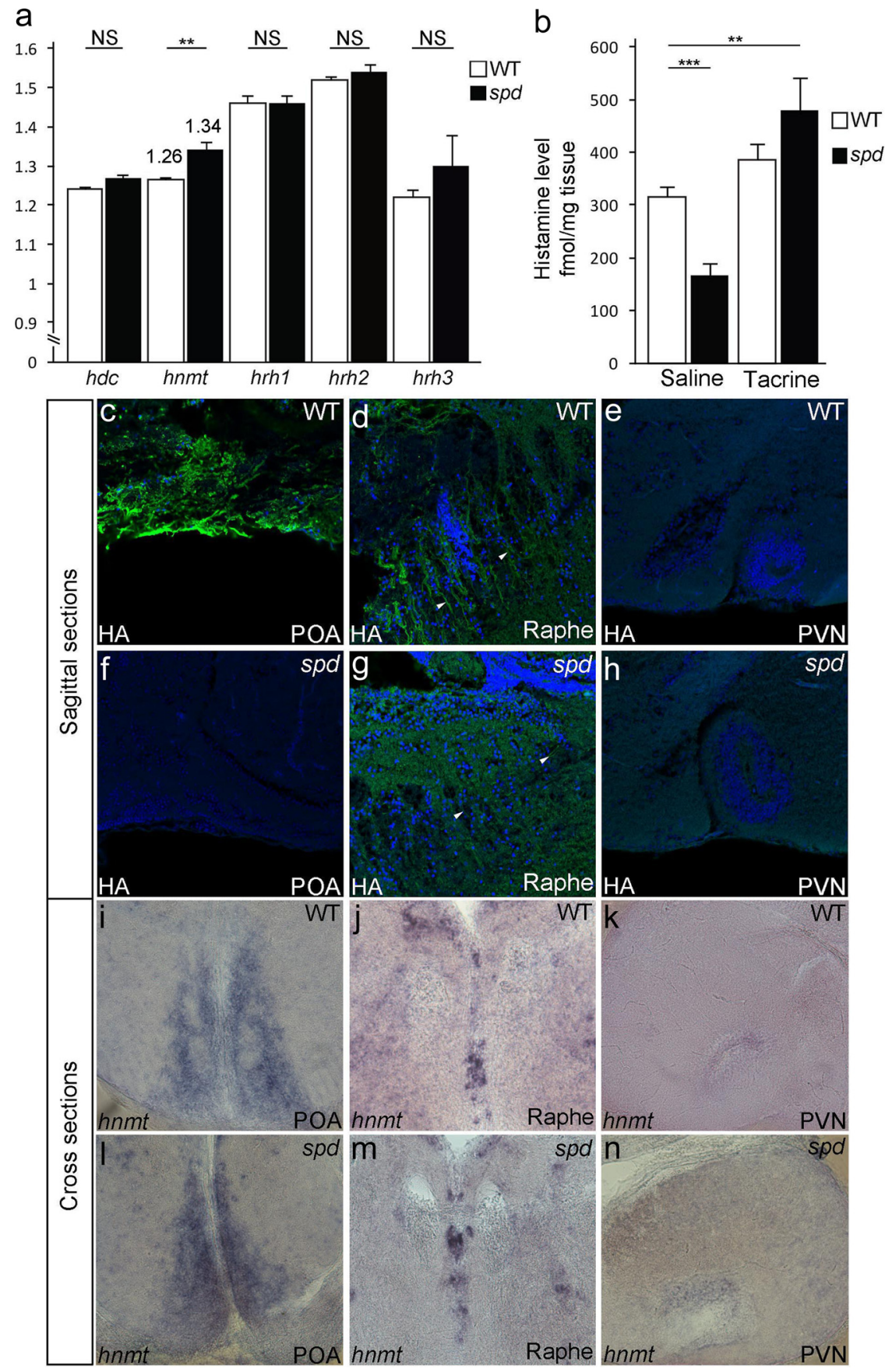

Figure 6. spd mutants have reduced histaminergic signaling in the brain. $\boldsymbol{a}$, Bar chart showing relative expression levels of histaminergic pathway genes in wild-type (white bars) and spd (black bars) brains. hnmt expression is significantly increased in spd compared with wild-type, whereas other genes are unaffected. $h d c, p=0.34 ; h n m t, p=0.007 ; h r h 1, p=$ $0.97 ; h r h 2, p=0.48$; and $h r h 3, p=0.63 ; n=10$ brains for each genotype. Expression ratio calculated relative to neurod followed by $t$ test. The number above the bars depicting wild-type and spd hnmt show the exact expression level of this gene to aid comparison. $\boldsymbol{b}$, Radioimmunoassay comparing levels of histamine in the brains of wild-type and spd, with and without tacrine treatment. Histamine is significantly reduced in spd compared with wild-type. Tacrine treatment increase histamine levels in spd but not wild-type. Wild-type versus spd, $F=25.13, p=0.0001$; tacrine versus salt, $F=9.83, p=$ $0.0064 ; n=5$ brains for each genotype and treatment. Two-way ANOVA without interaction. $\boldsymbol{c}-\boldsymbol{h}$, Anti-histamine antibody labeling is reduced in the brain of spd compared with wild-type. Sagittal sections showing histamine antibodypositive fibers in green and DAPI in blue. spd mutants have a reduction of histamine in the POA $(\boldsymbol{c}, \boldsymbol{f})$ and raphe nucleus ( $\boldsymbol{d}$, $\boldsymbol{g})$. No HA-positive fibers are seen in the PVN $(\boldsymbol{e}, \boldsymbol{h})$. White arrowheads show histamine-positive fibers in the raphe nucleus of both genotypes. The other green areas in the background of the image correspond to background in the antibody staining. $\boldsymbol{i}-\boldsymbol{n}, \boldsymbol{h n m t}$ is similarly expressed in the brain of wild-type and spd. Cross-sections showing expression in the POA $(\boldsymbol{i}, \boldsymbol{I})$, raphe nucleus $(\boldsymbol{j}, \boldsymbol{m})$, and PVN $(\boldsymbol{k}, \boldsymbol{n})$. Raphe, Raphe nucleus.
$6 i-n)$. This is in keeping with the widespread projection pattern of histaminergic neurons, whose cell-bodies are clustered in the Hc (Eriksson et al., 1998). Although the fold change of hnmt RNA was very small, it prompted us to investigate whether spd mutants have an imbalance in histaminergic neurotransmitter signaling. Analysis of neurotransmitter levels by radioimmunoassay (Fig. 6b) and with an HAspecific antibody (Fig. $6 c-h$ ) confirmed the reduction of $\mathrm{HA}$ in the mutant brain. The decrease in antibody labeling was seen in all brain areas, although a few HA-positive fibers were still seen in the raphe nucleus of spd (Fig. 6d,g). We reasoned that, if decreased HA was a relevant downstream component of impaired Fgf signaling, increasing HA levels should rescue some aspects of the spd phenotype. We treated homozygous spd mutants with a nonsedative dose (see Fig. $8 b$ ) of the Hnmt inhibitor tacrine hydrochloride (Horton et al., 2005) and observed an increase in HA levels in the brain (Fig. 6b). We then measured aggression, boldness, and exploration of tacrinetreated fish. Treatment with tacrine did not affect aggression or novel-object boldness levels in wild type, but reduced aggression and novel-object boldness in homozygous spd (Fig. 7a,b). Tacrine treatment had a weaker effect on place-preference boldness, only causing a nonsignificant trend reduction of boldness in spd (Fig. 8e). Thus, the novel-object boldness test appeared to be more sensitive to changes in HA level, and so we used it in our subsequent pharmacological experiments. Tacrine treatment increased the amount of time needed to explore the T-shaped tank in both wild-type and homozygous spd mutants, bringing both genotypes to the same exploration level (Fig. 7c). At low concentrations, tacrine hydrochloride is specific for $h n m t$, whereas at high concentrations it can also inhibit the acetylcholine breakdown enzyme acetylcholinesterase (Horton et al., 2005). To rule out a contribution of acetylcholine signaling to the spd behavioral phenotype, we treated homozygous spd mutants with a nonsedative dose (Fig. $8 c$ ) of the acetylcholinesterase inhibitor galantamine hydrochloride (Houghton and Howes, 2005) and measured aggression. Treatment with galantamine did not affect the aggression levels of either wild-type or mutant fish (Fig. $8 f$ ), ruling out a contribution of acetylcholine signaling to the aggressive behavior we observed.

In zebrafish, histaminergic neurotransmitter signaling is transduced by three receptors encoded by the genes hrh1, hrh2, and hrh3 (Peitsaro et al., 2007). All three 
genes are expressed at similar levels throughout the brain of wild-type and homozygous spd mutant fish. In the PVN, only $h r h 3$ expression was easily detectable, whereas $h r h 1$ was absent and hrh2 was only weakly expressed. To test whether the HA-linked behavior changes could be mediated by the histamine $\mathrm{H} 3$ receptor (Hrh3), we treated fish of both genotypes with a nonsedative dose (Fig. $8 d$ ) of the selective Hrh3 agonist imetit dihydrobromide (Garbarg et al., 1992). We measured aggression, novel-object boldness, and exploratory activity of imetit-treated fish. Treatment with imetit rescued the behavioral phenotype of homozygous spd mutants. Both aggression and novel-object boldness were reduced compared with wild-type siblings (Fig. $7 d, e$ ), whereas the amount of time needed to explore a $\mathrm{T}$-shaped aquarium was increased to the level of wild-type fish (Fig. $7 f$ ). Therefore, the combined results from these experiments suggest that modulation of HAergic signaling can rescue the Fgfr ladependent aggression-boldness phenotype observed in homozygous spd mutants.

\section{Discussion}

In this study, we have analyzed spiegeldanio mutant fish that have a reduction of fgfrla activity in the brain. Homozygous spd mutants show increases in aggression, boldness, and exploration, three components of the aggression-boldness behavioral syndrome. These behaviors are related to a reduction in histamine neurotransmitter levels in spd, and pharmacological manipulation of HA signaling is sufficient to rescue the observed behavioral defects.

Although behavioral syndromes have been extensively studied, the mechanism underlying their formation is not well understood. Behavioral syndromes could be caused by either genetic or hormonal constraint (Koolhaas et al., 1999; Drent et al., 2003; Veenema et al., 2003; Dingemanse et al., 2007) or by the selection of behaviors in response to environmental pressure (Bell, 2005; Bell and Sih, 2007). In this study, we have shown that polymorphism of a single gene, $f g f r 1 a$, is sufficient to induce parallel changes in three behaviors providing evidence that genetic constraint can influence the aggression-boldness behavioral syndrome. However, in another fish, the stickleback, the correlation between aggression and boldness varies depending on the testing environment (Bell, 2005; Bell and Sih, 2007). It thus seems likely that a combination of genetic or hormonal constraint and environmental conditions controls the expression of behavioral syndromes. Aggression is a malleable behavior, whereas boldness tends to be a more stable trait (Bell and Sih, 2007). Although our behavioral measurements were highly reproducible, we saw differences in aggression levels between zebrafish measured in different locations. Fish raised in Munich, Germany, were significantly more aggressive than those raised in

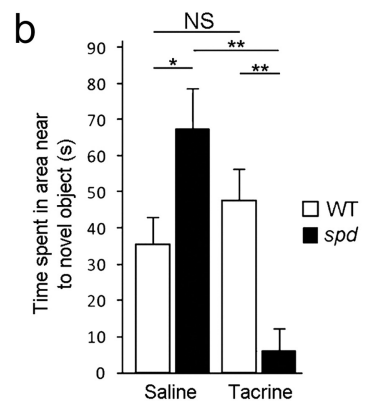

C
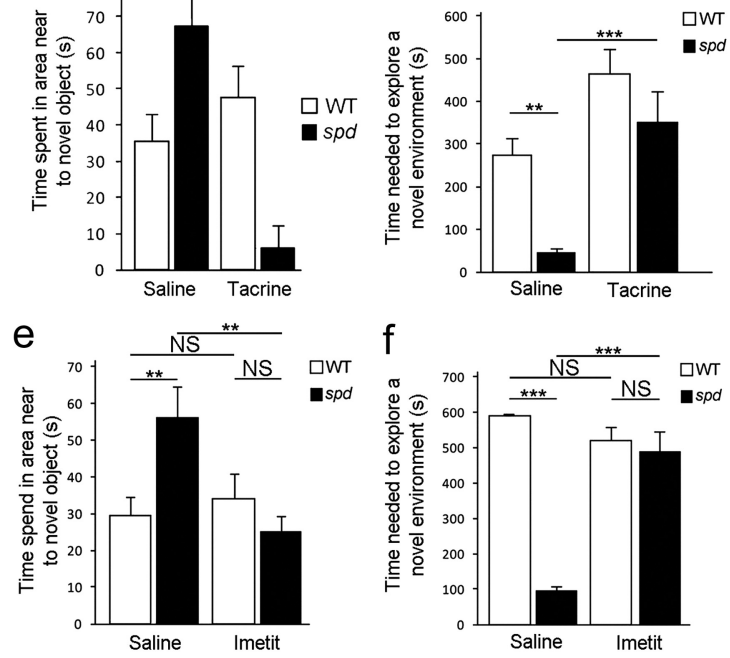

Figure 7. Changes in histaminergic signaling may underlie the aggression-boldness behavioral syndrome. $\boldsymbol{a}$, Treatment of $s p d$ pe versus spd saline, $p=0.02$; wild-type versus spd tacrine, $p=0.0004$; wild-type versus wild-type tacrine, $p=0.9$; spd versus $t$ test. $\boldsymbol{b}$. Treatment of spd with the hnmt inhibitor tacrine hydrochloride decreases boldness in spd (black bars) but not wild-type versus spd, $F=10.55, p=0.003$; saline versus tacrine, $F=22.29, p=0.001 ; n=10$ wild-type saline, $n=12$ spd saline, $n=$ the tacrine, and $n=10$ spd tacrine. Tw0-way ANOVA without interaction. $d$, Treatment of spd with the histamine $H 3$ spd saline $p=0.00007$ : wild-type versus spd imetit, $p=0.3 \cdot$ wild-type versus wild-type imetit, $p=0.07 \cdot$ spd versus spd imetit, $p=0.03 ; n=12$ wild-type saline, $n=10$ spd saline, $n=8$ wild-type imetit, and $n=8$ spd Imetit. ANOVA shows significant so both genotype effects were tested separately with a $t$ test. $e$ Treatment of spd with the histamine $\mathrm{H} 3$ receptor ; $p=0.02 ; n=12$ wild-type saline, $n=12$ spd saline, $n=12$ wild-type Imetit, and $n=12$ spd Imetit. ANOVA shows significant interaction, so both genotype effects were tested separately with a $t$ test. $\boldsymbol{f}$, Treatment of spd with the histamine $\mathrm{H} 3$ receptor saline $p=0.00001$. wild-type versus spd imetit $p=0.64$; wild-type versus wild-type imetit $p=0.1 \cdot$ spd versus spd imetit $p=$ 0.001. $n=10$ wild-type saline, $n=10$ spd saline, $n=10$ wild-type imetit, and $n=10$ spd imetit. ANOVA shows significant interaction, so both genotype effects were tested separately with a $t$ test.

Gif-sur-Yvette, France. Nevertheless, spd were always more aggressive than wild-type regardless of testing location (Fig. 9a). Zebrafish aggression levels can be affected by the environmental conditions during development (Marks et al., 2005), and behavioral assays are sensitive to changes in feeding regimen and diet, lighting conditions, $\mathrm{pH}$, time of day, and experimental observer (Wahlsten et al., 2006; Burgess and Granato, 2008). Although we made an effort to maintain similar conditions between laboratories, we cannot rule out the contribution of one (or all) of these variables to the alterations in aggression levels. Conversely, the boldness levels of both wild-type and spd were similar in both locations (Fig. 9b). Therefore, if the correlation between aggression and boldness varies in response to environmental factors, it may be the aggression component of the syndrome that is more likely to be modified.

Although spd mutant fish have a life-long reduction of Fgf signaling, it does not appear to adversely affect their lifespan, at least in the laboratory. Reduction of $f g f r 1 a$ function therefore appears to occur within an acceptable limit of developmental plasticity (West-Eberhard, 2005): adult behavioral changes occur 
a
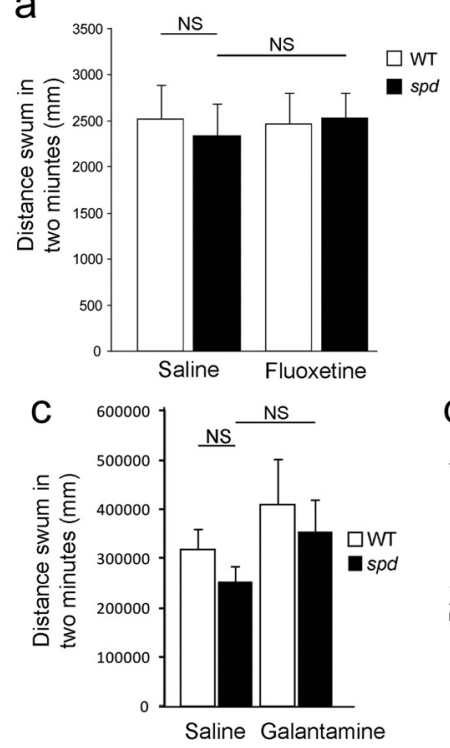

e

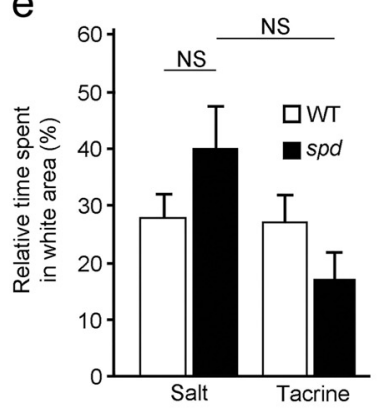

b

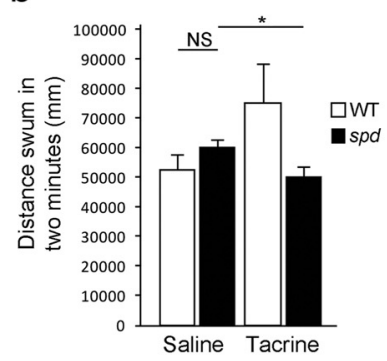

d

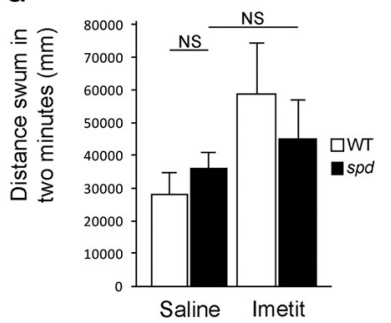

f

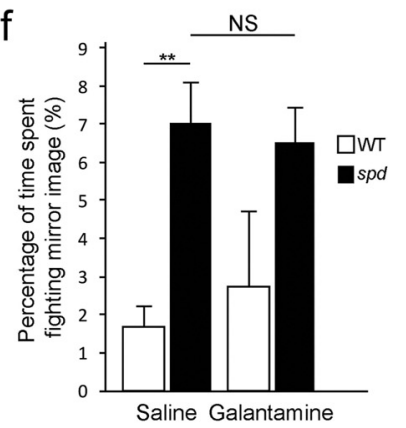

Figure 8. Control behavior of drug treated fish. $\boldsymbol{a}$, Fluoxetine hydrochloride does not affect locomotion levels of spd (black bars) or wild-type (white bars) fish in a familiar environment. Wild-type versus spd, $F=0.032, p=0.857$; fluoxetine versus saline, $F=0.056, p=0.832$; $n=6$ wild-type saline, $n=7$ wild-type fluoxetine, $n=8 \mathrm{spd}$ saline, and $n=6 \mathrm{spd}$ fluoxetine. Two-way ANOVA without interaction. $\boldsymbol{b}$, Tacrine hydrochloride does not affect the locomotion levels of spd (black bars) or wild-type (white bars) fish in a familiar environment. Wild-type versus spd, $F=0.75, p=0.4$; tacrine versus saline, $F=4.71, p=0.04 ; n=6$ wild-type saline, $n=6$ wild-type tacrine, $n=6$ spd saline, and $n=6$ spd tacrine. Two-way ANOVA without interaction. c, Galantamine hydrochloride does not affect the locomotion levels of spd (black bars) or wild-type (white bars) fish in a familiar environment. Wild-type versus spd, $F=0.74$, $p=0.4$; galantamine versus saline, $F=1.23, p=0.28 ; n=6$ wild-type saline, $n=6$ wild-type galantamine, $n=6 \mathrm{spd}$ saline, and $n=6 \mathrm{spd}$ galantamine. Two-way ANOVA without interaction. $\boldsymbol{d}$, Imetit dihydrobromide does not affect the locomotion levels of spd (black bars) or wild-type (white bars) fish in a familiar environment. Wild-type versus spd, $F=3.36$, $p=0.08$; imetit versus saline, $F=0.07, p=0.8 ; n=6$ wild-type saline, $n=6$ wild-type imetit, $n=6 \mathrm{spd}$ saline, and $n=6 \mathrm{spd}$ imetit. Two-way ANOVA without interaction. $\boldsymbol{e}$, Treatment with the hnmt inhibitor tacrine hydrochloride causes a nonsignificant reduction in boldness in spd (black bars) but not wild-type (white bars). Wild-type versus spd, $F=0.56, p=$ 0.45 ; saline versus tacrine, $F=2.133, p=0.15 ; n=15$ wild-type saline, $n=13$ spd saline, $n=12$ wild-type tacrine, and $n=9$ spd tacrine. Two-way ANOVA without interaction. $f$, Treatment of spd (black bars) with the acetylcholinesterase inhibitor galantamine hydrochloride does not decrease aggression in spd (black bars) or wild-type (white bars) fish. Wild-type versus spd, $F=9.09, p=0.0046$; galantamine versus saline, $F=0.09, p=0.77 ; n=10$ wild-type saline, $n=12$ spd saline, $n=8$ wild-type galantamine, and $n=12$ spd galantamine. Two-way ANOVA without interaction.

in the absence of deleterious developmental phenotypes. In this study, we have shown that mutation of fgfrla increases the number of zebrafish expressing aggression at the higher end of the natural spectrum (Fig. 2a). Although increased aggression is sometimes viewed as a negative trait, this result suggests that the $s p d$ behavioral phenotype may rather represent an extreme example of normal behavior (Garrod, 1902).
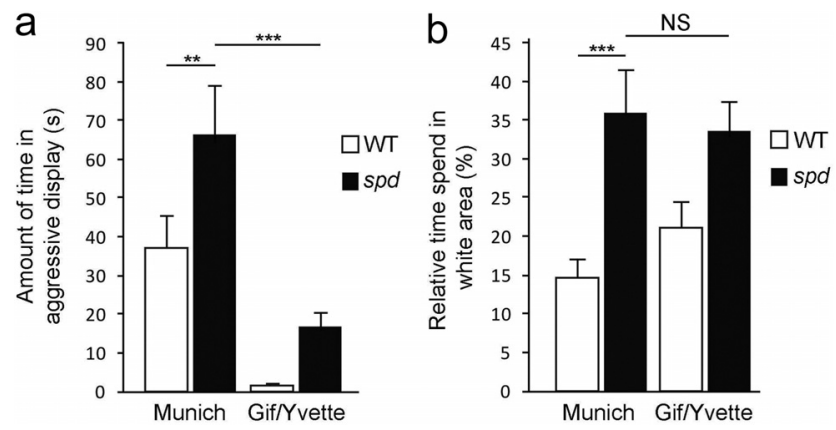

Figure 9. Comparison of the behavior of fish tested in two locations. $\boldsymbol{a}$, Zebrafish raised and analyzed in Gif-sur-Yvette, France, were less aggressive than fish raised and analyzed in Munich, Germany, regardless of genotype. However, spd mutant fish were more aggressive than wild-types in both locations. Munich fish versus Gif-sur-Yvette fish, $F=5.62, p=0.02$; wildtype versus spd, $F=22.23, p=0.001 ; n=31$ Munich wild-type, $n=27$ Munich spd, $n=20$ Gif-sur-Yvette wild-type, and $n=23$ Gif-sur-Yvette spd. Two-way ANOVA without interaction. $\boldsymbol{b}$, Zebrafish raised and analyzed in Gif-sur-Yvette, France, showed similar boldness levels as fish raised and analyzed in Munich, Germany. spd mutant fish were bolder than wild-types in both locations. Munich fish versus Gif-sur-Yvette fish, $F=0.29, p=0.59$; wild-type versus spd, $F=$ $13.89, p=0.008 ; n=6$ Munich wild-type, $n=7$ Munich spd, $n=10$ Gif-sur-Yvette wild-type, and $n=11$ Gif-sur-Yvette spd. Two-way ANOVA without interaction.

spiegeldanio mutants harbor a mutation in $f g f r l a$ that is a receptor for Fgfs, a large family of secreted signaling molecules with important roles during development (Thisse and Thisse, 2005). Zebrafish have multiple Fgf receptors that are coexpressed in the adult brain. For example, $f g f r 1 a$, $f g f r 2$, and $f g f r 3$ are all expressed in the PVN, central nucleus, and diffuse nucleus of the hypothalamus (Topp et al., 2008). Both $f g f r 2$ and $f g f r 3$ are expressed normally throughout the brain of homozygous spd mutants. The most obvious reduction of Fgf signaling in spd is found in the PVN. The PVN has already been associated with the control of aggression in teleosts. Electrical stimulation of the nucleus of the lateral recess of the third ventricle (equivalent to the zebrafish PVN) causes aggressive interaction with a mirror in the Bluegill (Demski and Knigge, 1971). However, additional brain areas may also play an important role in linking aggression, boldness, and exploration. For example, a recent gene expression analysis of aggressive zebrafish identified gene expression changes in the telencephalon, optic tectum, hypothalamus, and hindbrain (Filby et al., 2010). It is clear that an in-depth analysis of the spd mutant brain is needed to resolve this issue.

Our analysis of $s p d$ mutants demonstrates that a reduction of Fgfr la signaling results in increased expression of the HA breakdown enzyme-encoding gene $h n m t$, correlating with decreased levels of HA in the brain. Although hnmt expression was only marginally increased in spd, modulation of HA levels was sufficient to reverse the behavioral phenotype. However, such a slight change in the mRNA expression level of hnmt is unlikely to fully explain the changes observed. Thus, an additional (unidentified) mechanism may also disrupt HA levels in spd. Alternatively, the upregulation of hnmt may only occur in some discrete areas of the mutant brain. Our analysis of hnmt levels in whole zebrafish brain extracts precludes us from ruling out this possibility.

In zebrafish, similar to other animals, HA neurons are found exclusively in the caudal hypothalamus and send projections to many brain areas (Eriksson et al., 1998). We observed a strong reduction of HA-positive fibers throughout the brain of spd (Fig. $6 c-h$ ). Although the PVN (the area of the brain with the most striking reduction of Fgf signaling) does not produce HA, it is positive for both hnmt expression and the histamine receptor- 
encoding genes $h r h 2$ and $h r h 3$ at weak and strong levels, respectively. Rescue of the behavioral phenotype of $s p d$ mutants with the Hrh3 agonist imetit (Garbarg et al., 1992) provides evidence that Hrh3 may underlie these behaviors, although we cannot explicitly rule out a contribution of Hrh1 and Hrh2 activity as well. Hrh3 acts as both an autoreceptor and a heteroreceptor (Peitsaro et al., 2000) and has a complex role in the control of HA neurotransmission (Leurs et al., 2005; Jones and Kearns, 2011). Hrh3 knock-out mice have a net decrease of HA levels in the cortex (Toyota et al., 2002) and show reduced anxiety in an elevated plus maze (Rizk et al., 2004) reminiscent of the spd phenotype. Histamine has been linked previously to several behaviors, including aggression, exploration, and boldness (Lecklin et al., 1998; Yanai et al., 1998; Peitsaro et al., 2003; Passani et al., 2004; Masaki and Yoshimatsu, 2006). Thus, HA may have a role as a general regulator of brain function rather than influencing specific behaviors (Wada et al., 1991).

To our knowledge, there are currently no studies linking Fgf and HA, making it difficult to understand how these signaling pathways might interact. However, our data suggest two possible mechanisms. Fgf signaling could act on hnmt expression independently of HA, reducing the activity of the enzyme. A reduction of $f g f r 1 a$ in spd mutants would lead to a concomitant increase in Hnmt activity and HA turnover. Alternatively, Fgfrla could interact with Hrh3 signaling at the level of MAPK signaling. Stimulation of Hrh3 receptors results in MAPK activation (Drutel et al., 2001), providing a possible substrate for interaction with Fgf that also uses MAPK as a target (Thisse and Thisse, 2005). Reduction of fgfrla may decrease the sensitivity of Hrh3-expressing cells to Hrh 3 activation. Because we only observe a slight increase in hnmt expression levels in mutants, we tend to favor this second hypothesis. Together, our results suggest that Fgf signaling may sensitize the brain to changes in HAegic signaling levels, thus altering the expression of the aggression-boldness behavior syndrome. Nevertheless, it is important to remember that spd mutants have a life-long reduction of Fgf signaling, meaning that the behavioral phenotype we observe may stem from changes in brain development. It is therefore likely that other neurotransmitter pathways may also mediate aspects of the spd behavioral phenotype. Additional studies will be required to address this issue.

\section{References}

Basquill SP, Grant JWA (1997) An increase in habitat complexity reduces aggression and monopolization of food by zebrafish (Danio rerio). Can J Zool 76:770-772.

Bell AM (2005) Behavioural differences between individuals and two populations of stickleback (Gasterosteus aculeatus). J Evol Biol 18:464-473.

Bell AM, Sih A (2007) Exposure to predation generates personality in threespined sticklebacks (Gasterosteus aculeatus). Ecol Lett 10:828-834.

Benus RF, Bohus B, Koolhaas JM, van Oortmerssen GA (1991) Heritable variation for aggression as a reflection of individual coping strategies. Experientia 47:1008-1019.

Boissy A (1995) Fear and fearfulness in animals. Q Rev Biol 70:165-191.

Bourne GR, Sammons AJ (2008) Boldness, aggression and exploration: evidence for a behavioural syndrome in male pentamorphic livebearing fish, Poecilia parae. AACL Bioflux 1:39-49.

Brick O, Jakobsson S (2001) Individual variation in risk taking: the effect of a predatory threat on fighting behavior in Nannacara anomola. Behav Ecol 13:439-442.

Burgess HA, Granato M (2008) The neurogenetic frontier-lessons from misbehaving zebrafish. Brief Funct Genomic Proteomic 7:474-482.

Clotfelter ED, O'Hare EP, McNitt MM, Carpenter RE, Summers CH (2007) Serotonin decreases aggression via 5-HT1A receptors in the fighting fish Betta splendens. Pharmacol Biochem Behav 87:222-231.

Demski LS, Knigge KM (1971) The telencephalon and hypothalamus of the bluegill (Lepomis macrochirus): evoked feeding, aggressive and reproductive behavior with representative frontal sections. J Comp Neurol 143: $1-16$.

Deng CX, Wynshaw-Boris A, Shen MM, Daugherty C, Ornitz DM, Leder P (1994) Murine FGFR-1 is required for early postimplantation growth and axial organization. Genes Dev 8:3045-3057.

Dingemanse NJ, Wright J, Kazem AJ, Thomas DK, Hickling R, Dawnay N (2007) Behavioural syndromes differ predictably between 12 populations of three-spined stickleback. J Anim Ecol 76:1128-1138.

Dodé C, Levilliers J, Dupont JM, De Paepe A, Le Dû N, Soussi-Yanicostas N, Coimbra RS, Delmaghani S, Compain-Nouaille S, Baverel F, Pêcheux C, Le Tessier D, Cruaud C, Delpech M, Speleman F, Vermeulen S, Amalfitano A, Bachelot Y, Bouchard P, Cabrol S, et al. (2003) Loss-of-function mutations in FGFR1 cause autosomal dominant Kallmann syndrome. Nat Genet 33:463-465.

Drent PJ, van Oers K, van Noordwijk AJ (2003) Realized heritability of personalities in the great tit (Parus major). Proc Biol Sci 270:45-51.

Drutel G, Peitsaro N, Karlstedt K, Wieland K, Smit MJ, Timmerman H, Panula P, Leurs R (2001) Identification of rat $\mathrm{H} 3$ receptor isoforms with different brain expression and signaling properties. Mol Pharmacol 59:1-8.

Eriksson KS, Peitsaro N, Karlstedt K, Kaslin J, Panula P (1998) Development of the histaminergic neurons and expression of histidine decarboxylase mRNA in the zebrafish brain in the absence of all peripheral histaminergic systems. Eur J Neurosci 10:3799-3812.

Filby AL, Tyler CR (2005) Molecular characterization of estrogen receptors $1,2 \mathrm{a}$, and $2 \mathrm{~b}$ and their tissue and ontogenic expression profiles in fathead minnow (Pimephales promelas). Biol Reprod 73:648-662.

Filby AL, Paull GC, Hickmore TF, Tyler CR (2010) Unravelling the neurophysiological basis of aggression in a fish model. BMC Genomics 11:498.

Garbarg M, Arrang JM, Rouleau A, Ligneau X, Tuong MD, Schwartz JC, Ganellin CR (1992) S-[2-(4-imidazolyl)ethyl] isothiourea, a highly specific and potent histamine H3 receptor agonist. J Pharmacol Exp Ther 263:304-310.

Garrod AE (1902) About Alkaptonuria. Med Chir Trans 85:69-78.

Gerlai R, Lahav M, Guo S, Rosenthal A (2000) Drinks like a fish: zebra fish (Danio rerio) as a behavior genetic model to study alcohol effects. Pharmacol Biochem Behav 67:773-782.

Gross C, Zhuang X, Stark K, Ramboz S, Oosting R, Kirby L, Santarelli L, Beck S, Hen R (2002) Serotonin1A receptor acts during development to establish normal anxiety-like behaviour in the adult. Nature 416:396-400.

Haas HL, Sergeeva OA, Selbach O (2008) Histamine in the nervous system. Physiol Rev 88:1183-1241.

Holm S (1979) A simple sequentially rejective multiple test procedure. Scand J Stat 6:65-70.

Horton JR, Sawada K, Nishibori M, Cheng X (2005) Structural basis for inhibition of histamine $\mathrm{N}$-methyltransferase by diverse drugs. J Mol Biol 353:334-344.

Houghton PJ, Howes MJ (2005) Natural products and derivatives affecting neurotransmission relevant to Alzheimer's and Parkinson's disease. Neurosignals 14:6-22.

Huntingford FA (1976) The relationship between anti-predator behaviour and aggression among conspecifics in the three-spined stickleback, Gasterosteus aculeatus. Anim Behav 24:245-260.

Jones BL, Kearns GL (2011) Histamine: new thoughts about a familiar mediator. Clin Pharmacol Ther 89:189-197.

Koolhaas JM, Korte SM, De Boer SF, Van Der Vegt BJ, Van Reenen CG, Hopster H, De Jong IC, Ruis MA, Blokhuis HJ (1999) Coping styles in animals: current status in behavior and stress-physiology. Neurosci Biobehav Rev 23:925-935.

Larson ET, O’Malley DM, Melloni RH Jr (2006) Aggression and vasotocin are associated with dominant-subordinate relationships in zebrafish. Behav Brain Res 167:94-102.

Lecklin A, Etu-Seppälä P, Stark H, Tuomisto L (1998) Effects of intracerebroventricularly infused histamine and selective $\mathrm{H} 1, \mathrm{H} 2$ and $\mathrm{H} 3$ agonists on food and water intake and urine flow in Wistar rats. Brain Res 793:279-288.

Leurs R, Bakker RA, Timmerman H, de Esch IJ (2005) The histamine H3 receptor: from gene cloning to $\mathrm{H} 3$ receptor drugs. Nat Rev Drug Discov 4:107-120.

Livak KJ, Schmittgen TD (2001) Analysis of relative gene expression data 
using real-time quantitative PCR and the 2(-Delta Delta $\mathrm{C}(\mathrm{T}))$ method. Methods 25:402-408.

Lynn SE, Egar JM, Walker BG, Sperry TS, Ramenofsky M (2007) Fish on Prozac: a simple, noninvasive physiology laboratory investigating the mechanisms of aggressive behavior in Betta splendens. Adv Physiol Educ 31:358-363.

Marks C, West TN, Bagatto B, Moore FBG (2005) Developmental environment alters conditional aggression in zebrafish. Copeia 4:901-908.

Masaki T, Yoshimatsu H (2006) The hypothalamic H1 receptor: a novel therapeutic target for disrupting diurnal feeding rhythm and obesity. Trends Pharmacol Sci 27:279-284.

Moretz JA, Martins EP, Robison BD (2007) Behavioural syndromes and the evolution of correlated behavior in zebrafish. Behav Ecol 18:556-562.

Morisset S, Traiffort E, Schwartz JC (1996) Inhibition of histamine versus acetylcholine metabolism as a mechanism of tacrine activity. Eur J Pharmacol 315:R1-R2.

Ninkovic J, Bally-Cuif L (2006) The zebrafish as a model system for assessing the reinforcing properties of drugs of abuse. Methods 39:262-274.

Norton WH, Folchert A, Bally-Cuif L (2008) Comparative analysis of serotonin receptor (HTR1A/HTR1B families) and transporter (slc6a4a/b) gene expression in the zebrafish brain. J Comp Neurol 511:521-542.

Passani MB, Lin JS, Hancock A, Crochet S, Blandina P (2004) The histamine $\mathrm{H} 3$ receptor as a novel therapeutic target for cognitive and sleep disorders. Trends Pharmacol Sci 25:618-625.

Peitsaro N, Anichtchik OV, Panula P (2000) Identification of a histamine $\mathrm{H}(3)$-like receptor in the zebrafish (Danio rerio) brain. J Neurochem 75:718-724.

Peitsaro N, Kaslin J, Anichtchik OV, Panula P (2003) Modulation of the histaminergic system and behaviour by alpha-fluoromethylhistidine in zebrafish. J Neurochem 86:432-441.

Peitsaro N, Sundvik M, Anichtchik OV, Kaslin J, Panula P (2007) Identification of zebrafish histamine $\mathrm{H} 1, \mathrm{H} 2$ and $\mathrm{H} 3$ receptors and effects of histaminergic ligands on behavior. Biochem Pharmacol 73:1205-1214.

Perreault HA, Semsar K, Godwin J (2003) Fluoxetine treatment decreases territorial aggression in a coral reef fish. Physiol Behav 79:719-724.

Ramboz S, Saudou F, Amara DA, Belzung C, Segu L, Misslin R, Buhot MC, Hen R (1996) 5-HT1B receptor knock out-behavioral consequences. Behav Brain Res 73:305-312.

Réale D, Reader SM, Sol D, McDougall PT, Dingemanse NJ (2007) Integrating animal temperament within ecology and evolution. Biol Rev Camb Philos Soc 82:291-318.

Rizk A, Curley J, Robertson J, Raber J (2004) Anxiety and cognition in histamine H3 receptor - / - mice. Eur J Neurosci 19:1992-1996.

Rohner N, Bercsényi M, Orbán L, Kolanczyk ME, Linke D, Brand M, Nüsslein-Volhard C, Harris MP (2009) Duplication of fgfr1 permits Fgf signaling to serve as a target for selection during domestication. Curr Biol 19:1642-1647.

Rowland WJ (1999) Studying visual cues in fish behaviour: a review of ethological techniques. Environ Biol Fishes 56:285-305.
Schjolden J, Backström T, Pulman KG, Pottinger TG, Winberg S (2005) Divergence in behavioural responses to stress in two strains of rainbow trout (Oncorhynchus mykiss) with contrasting stress responsiveness. Horm Behav 48:537-544.

Sih A, Bell A, Johnson JC (2004a) Behavioral syndromes: an ecological and evolutionary overview. Trends Ecol Evol 19:372-378.

Sih A, Bell AM, Johnson JC, Ziemba RE (2004b) Behavioral syndromes: an intergrative overiew. Q Rev Biol 79:241-277.

Thisse B, Thisse C (2005) Functions and regulations of fibroblast growth factor signaling during embryonic development. Dev Biol 287:390-402.

Topp S, Stigloher C, Komisarczuk AZ, Adolf B, Becker TS, Bally-Cuif L (2008) Fgf signaling in the zebrafish adult brain: association of Fgf activity with ventricular zones but not cell proliferation. J Comp Neurol 510:422-439.

Toyota H, Dugovic C, Koehl M, Laposky AD, Weber C, Ngo K, Wu Y, Lee DH, Yanai K, Sakurai E, Watanabe T, Liu C, Chen J, Barbier AJ, Turek FW, FungLeung WP, Lovenberg TW (2002) Behavioral characterization of mice lacking histamine $\mathrm{H}(3)$ receptors. Mol Pharmacol 62:389-397.

Veenema AH, Meijer OC, de Kloet ER, Koolhaas JM (2003) Genetic selection for coping style predicts stressor susceptibility. J Neuroendocrinol 15:256-267.

Vitebsky A, Reyes R, Sanderson MJ, Michel WC, Whitlock KE (2005) Isolation and characterization of the laure olfactory behavioral mutant in the zebrafish, Danio rerio. Dev Dyn 234:229-242.

Wada H, Inagaki N, Yamatodani A, Watanabe T (1991) Is the histaminergic neuron system a regulatory center for whole-brain activity. Trends Neurosci 14:415-418.

Wahlsten D, Bachmanov A, Finn DA, Crabbe JC (2006) Stability of inbred mouse strain differences in behavior and brain size between laboratories and across decades. Proc Natl Acad Sci USA 103:16364-16369.

West-Eberhard MJ (2005) Developmental plasticity and the origin of species differences. Proc Natl Acad Sci USA 102 [Suppl 1]:6543-6549.

Wolf M, van Doorn GS, Leimar O, Weissing FJ (2007) Life-history tradeoffs favour the evolution of animal personalities. Nature 447:581-584.

Wright D, Rimmer LB, Pritchard VL, Krause J, Butlin RK (2003) Inter and intra-population variation in shoaling and boldness in the zebrafish (Danio rerio). Die Naturwissenschaften 90:374-377.

Yanai K, Son LZ, Endou M, Sakurai E, Nakagawasai O, Tadano T, Kisara K, Inoue I, Watanabe T, Watanabe T (1998) Behavioural characterization and amounts of brain monoamines and their metabolites in mice lacking histamine $\mathrm{H} 1$ receptors. Neuroscience 87:479-487.

Yokoi H, Shimada A, Carl M, Takashima S, Kobayashi D, Narita T, Jindo T, Kimura T, Kitagawa T, Kage T, Sawada A, Naruse K, Asakawa S, Shimizu N, Mitani H, Shima A, Tsutsumi M, Hori H, Wittbrodt J, Saga Y, Ishikawa Y, Araki K, Takeda H (2007) Mutant analyses reveal different functions of fgfr 1 in medaka and zebrafish despite conserved ligand-receptor relationships. Dev Biol 304:326-337. 